\title{
Chair-rising and 3-min walk: A simple screening test for functional mobility
}

\author{
Tae-Woong Oh ${ }^{1 *}$, Izumi Tabata ${ }^{2}$, Jin-Hwan Kim ${ }^{3}$, Tae-Hyun Lee ${ }^{4}$, Tatsuki Naka ${ }^{5}$ \\ ${ }^{1}$ Graduate School of Health Science, Matsumoto University, Matsumoto, Japan; *Corresponding Author: taewoong@matsu.ac.jp \\ ${ }^{2}$ Graduate School of Sport and Health Science, Ritsumeikan University, Kusatsuki, Japan \\ ${ }^{3}$ College of Physical Education, Kei-Myung University, Dae-Gu, Korea \\ ${ }^{4}$ College of Martial Arts, Yong-In University, Yong-In, Korea \\ ${ }^{5}$ Department of Health \& Sports Science, Faculty of Health Science, Shigakkan University, Obu, Japan
}

Received 3 October 2011; revised 17 November 2011; accepted 12 December 2011

\section{ABSTRACT}

Aging induces decrease of locomotor capacity and its decrease is associated with an increased risk of falls. Several lines of evidence indicate that both change in muscle power and aerobic fitness are causative. Mobility tests are usually based on a maximal exercise stress test; however, this test is often difficult and sometimes frightening to older persons. Therefore, the objective of this study was to examine age and gender differences in 3-min walk distance test (3WDT), and time of chair-rising test (CRT) of functional mobility. 153 men and 159 women aged from 20 to 78 years were recruited as subjects of the present study. The body composition measured the height, body mass (BM), body mass index (BMI), lean tissue mass (LTM), and waist circumference (WC). The Functional mobility tests measured the peak oxygen uptake $\left(\mathrm{V}_{\text {o2peak }}\right)$, 3WDT, leg extension strength (LES), and times of CRT. Both in men and women, height and BMI, WC decreased and increased, respectively, with age. Height, BM, LTM, WC in men are higher than in women. We found no correlation between ages and 3WDT in women and a significant, negative correlation in men. All parameters of fitness performance were negatively correlated with age. Both in men and women, all parameters of fitness performance were positively correlated with sex. Both in men and women, $\mathrm{V}_{\text {O2peak, }}$ 3WDT, and LES decreased with age. All parameters of fitness performance in men are higher than in women. Both in men and women were observed for the correlation between 3WDT and $V_{\text {O2peak, LES and CRT respectively. }}$ Although as the correlation coefficient between 3WTD and $V_{\text {O2peak, }}$, LES and CRT were low ( $r=$ 0.28 - 0.38), an error may occur, this study shows that 3WDT and CRT test can be a feasible method of providing the information for muscle power and aerobic fitness, possibly avoiding the need for a maximal stress test.

Keywords: Peak Oxygen Uptake; 3-Min Walk Distance Test; Leg Extension Strength; Chair-Rising Test; Mobility

\section{INTRODUCTION}

Aging induces decrease of locomotor capacity and its decrease is associated with an increased risk of falls. Current demographic trends show that the number of older people is rapidly increasing. In fact, mobility is essential for functional independence, reduced risk of fall, and quality of life [1-3]. In older persons, disability is caused by both change in muscle power and aerobic fitness is causative. Several studies have shown that there is a decline in the ability to perform muscle power-related tests as age increases with a significant decline commencing at approximately 40 years of age. Similarly, physical performance decrease with age. These age-related changes in the performance of functional mobility measures and physiological domains are also associated with an increased risk of falls, ongoing disability and admission into residential aged care $[3,4]$.

Mobility tests are commonly used to assess function and frailty in older persons. Mobility tests are usually based on a maximal exercise stress test; however, this test is often difficult and sometimes frightening to older persons. 3-min walk distance test and chair-rising test are low of risk. There are little data available on the age-related changes and gender differences in the performance of these tests. The development of age stratified normative data for these commonly used functional mobility tests could assist in the targeting of interventions for people who exhibit a decline in their functional status at an early stage, prior to the occurrence of falls and the onset of disability. Therefore, the aim of this study was to provide reference data and ex- 
amine age and gender differences in 3-min walk distance test, and time of rising chair without using the arms. The second aim was to provide data available on the age-related changes in the performance of these tests. The information provided is relevant to new functional mobility tests in older persons.

\section{METHODS}

\subsection{Participants}

One hundred and fifty-three men and one hundred and fifty-nine women aged from 20 to 78 years $(44.3 \pm 14.8$ years) were recruited as subjects of the present study. None of the subjects had any chronic diseases or were taking any medications that could affect the study variables. All subjects provided written informed consent according to local institute policy before the measurement of physical fitness. All subjects were classified into six groups by sex and age: 20 to 39-year-old men, 40 to 59-year-old men, 60 to 79-year-old men, 20 to 39-year-old women, 40 to 59-year-old women, and 60 to 79-year-old women. This study has been approved by the Committee on the Use of Human Research Subjects of Matsumoto University, and also performed in accordance with the ethical standards of the IJSM [5]. Participants were fully informed of the purpose and risks of participating in this investigation and signed informed consent documents prior to testing. The participants characteristics are described in Table $\mathbf{1 .}$

\subsection{Anthropometrics}

The body composition measured the height, body mass (BM), body mass index (BMI), lean tissue mass (LTM), and waist circumference (WC). Height was measured to the nearest $0.1 \mathrm{~cm}$ using a stadiometer (YKH-23; Yagami Inc., Japan). BM, BMI, and LTM were measured using a body composition meter (BC-118E; TANITA Inc., Japan).

\subsection{Functional Mobility Tests}

The four tests were administered in a single session. Ti- med tests were measured with stopwatch with an accuracy of $0.01 \mathrm{~s}$.

\subsection{Leg Extension Strength (LES)}

LES was assessed using GT-330 (OG-giken, Japan). The individuals were seated in the chair of the dynamometer, and were stabilized with straps across the waist and thighs throughout the test. Bilateral reciprocal contractions at the knee were measured at a preset angle of $120^{\circ}$. An index of strength was determined by summing peak extension torque. The average value in two times the right and left was assumed to be measurements.

\subsection{Peak Oxygen Uptake ( $\left.\mathrm{V}_{\text {o2peak }}\right)$}

$\mathrm{V}_{\mathrm{O} \text { peak }}$ was measured using a maximal graded exercise test (GTX) with bicycle erometers (Monark Ergomedic 828E, Sweden). The initial workload was $30-60 \mathrm{~W}$, and the work rate was increased thereafter by $15 \mathrm{~W} \cdot \mathrm{min}^{-1}$ until subject could not maintain the required pedaling frequency (60 rpm). Heart rate (WEP-7404; NIHON KOHDEN Corp., Japan) and a rating of perceived exertion were monitored throughout the exercise. During the progressive exercise test, the expired gas of subjects was collected, and the rates of oxygen consumption and Carbon dioxide production were measured and averaged over 30-s intervals using an automated breath-by-breath gas analyzing system (Aeromonitor AE-280S; Minato Medical Science, Japan).

\subsection{Chair-Rising Test (CRT)}

In this test, participants were asked to rise from a standard height $(43 \mathrm{~cm})$ chair without armrests, ten times as fast as possible with their arms folded. Arms are crossed in front of the chest. Participants undertook the test barefoot. The time from the initial seated position to the final seated position after completing ten stands was the test measure. Two trials were to be performed. The higher value in two trials was assumed to be measurements.

Table 1. Physical characteristic of the study subjects, mean \pm SD.

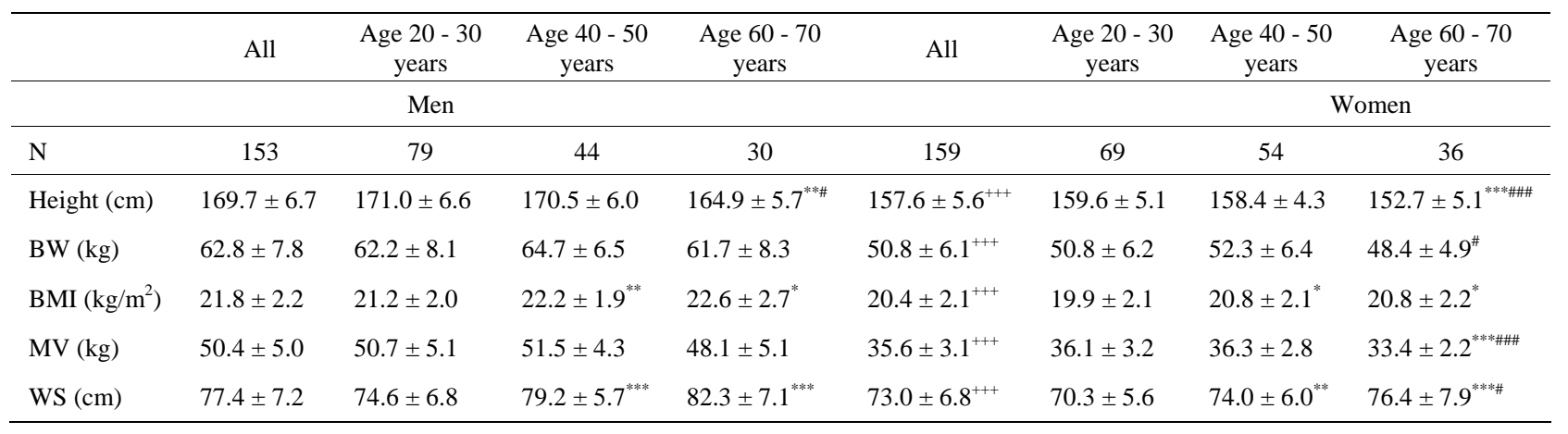

SD = Standard Deviation; BW = Body Weight; BMI = Body Mass Index; MV = Muscle Volume; Waist Size $=$ WS; ${ }^{* * *} \mathrm{p}<0.001,{ }^{* *} \mathrm{p}<0.01,{ }^{*} \mathrm{p}<0.05$ vs Age 20 - 30 years ${ }^{\# \#} \mathrm{p}<0.001,{ }^{\#} \mathrm{p}<0.01,{ }^{\#} \mathrm{p}<0.05$ vs Age $40-50$ years ${ }^{+++} \mathrm{p}<0.001$ vs men. 


\subsection{3-Min Walk Distance Test (3WDT)}

The participants performed the 3WDT in a 50-m indoor corridor with marks every second metre on the side of the walkway. They were instructed to wear comfortable shoes. The instructions were to walk as many lengths as possible in three minutes, without running or jogging. To clarify the instructions, the participants were also told to walk as fast as possible. Information was given during the test by telling the participants how many minutes they had walked or minutes remaining. Finally, the total 3WDT was measured.

\subsection{Statistical Analyses}

Results are expressed as mean values with their standard errors. The statistical significance (p, 0.05) of differences was determined by 2-way ANOVA followed by a Tukey post hoc analysis. Correlations between a fitness performance and another fitness performance were assessed by Pearson's correlation coefficients (r).

\section{RESULTS}

The physical characteristic of the study is described in Table 1. Height decreased and BMI, WC increased in men, respectively, with age. Height, BM and LTM decreased and BMI and WC increased in women. All physical characteristic in men are higher than in women. Table 2 reports the correlation between ages and functional mobility tests. All functional mobility tests, except for 3WDT in women, were negatively correlated with age (Table 2). Table 3 reports the parameters of functional mobility tests of the study subjects. All parameters of functional mobility tests were positively correlated with sex. All parameters of functional mobility tests in men are higher than in women. Both in men and women were observed for the correlation between 3WDT and $\mathrm{V}_{\text {O2peak }}$, LES and CRT respectively. Figure 1 reports the relationship between 3WDT and $\mathrm{V}_{\text {O2peak }}$ in the men $(\mathrm{n}=153)$ and women $(\mathrm{n}=159)$. Both in men and women, 3WTD was correlated with $\mathrm{V}_{\text {O2peak }}(\mathrm{r}$ $=0.31$ and 0.31, respectively; $\mathrm{p}<0.0001$ ). Figure 2 reports the relationship between LES and CRT in the men $(\mathrm{n}=153)$ and women $(\mathrm{n}=159)$. Both in men and women, LES was correlated with CRT $(r=0.38$ and 0.28 , respectively; $\mathrm{p}<0.001$ ).

\section{DISCUSSION}

3WDT and CRT is the simplest test of the $\mathrm{V}_{\text {O2peak }}$ test and leg strength, respectively. This study adds to the accumulating literature investigating the dynamic relations between body compositions and the functional mobility test in the elderly.

Body composition varies according to age, sex, and race. Older adults tend to lose fat-free mass and gain fat mass. WC is a reliable marker of mortality in older adults [6-8] and muscle mass, as represented by lean mass, is associated with survival. In the present study, height, BMI and WC were decreased and increased, respectively, with age in men and women. Moreover, Height, BM, LTM and WC in men is higher than in women.

The Functional mobility tests measured the $\mathrm{V}_{\text {O2peak, }}$ 3WDT, LES, and CRT. The study findings revealed significant age-related differences in all functional mobility tests examined. These findings confirm those of previous studies and indicate that when compared with young people, older people exhibit slower comfortable walking speed [5,9], reduced ability to quickly rise from a chair $[3,10]$. These age-related differences in functional mobility have been attributed to impaired sensorimotor function [11,12], in particular reduced lower extremity strength and power [13-15], but also increased fear of falling [8] and reduced aerobic capacity [16].

Table 2. Correlation of the variables of interest with age.

\begin{tabular}{ccccc}
\hline & CRT (sec) & 3WDT (m) & LE (n - m) & $\begin{array}{c}\mathrm{V}_{\text {O2max }} \\
(\mathrm{ml} / \mathrm{kg} / \mathrm{min})\end{array}$ \\
\hline Women & $0.19^{*}$ & -0.12 & $-0.32^{* *}$ & $-0.49^{* *}$ \\
Men & $0.42^{* *}$ & $-0.32^{* *}$ & $-0.32^{* *}$ & $-0.51^{* *}$ \\
\hline
\end{tabular}

CRT = chair-rising test; 3WDT = 3-min walk distance test; $\mathrm{LE}=$ leg extension; $\mathrm{V}_{\mathrm{O} 2 \max }=$ maximum oxygen uptake; Pearsons correlation coefficients. p $<0.001, \stackrel{*}{*}$ $<0.05$.

Table 3. Parameters of fitness performance of the study subjects, mean \pm SD.

\begin{tabular}{|c|c|c|c|c|c|c|c|c|}
\hline & All & $\begin{array}{c}\text { Age } 20-30 \\
\text { years }\end{array}$ & $\begin{array}{c}\text { Age } 40-50 \\
\text { years }\end{array}$ & $\begin{array}{c}\text { Age } 60-70 \\
\text { years }\end{array}$ & All & $\begin{array}{c}\text { Age } 20-30 \\
\text { years }\end{array}$ & $\begin{array}{c}\text { Age } 40-50 \\
\text { years }\end{array}$ & $\begin{array}{c}\text { Age } 60-70 \\
\text { years }\end{array}$ \\
\hline \multicolumn{6}{|c|}{ Men } & \multicolumn{3}{|c|}{ Women } \\
\hline $\mathrm{N}$ & 153 & 79 & 44 & 30 & 159 & 69 & 54 & 36 \\
\hline $\begin{array}{c}\mathrm{V}_{\mathrm{O} 2 \max } \\
(\mathrm{ml} / \mathrm{kg} / \mathrm{min})\end{array}$ & $36.7 \pm 9.1$ & $40.7 \pm 8.1$ & $35.0 \pm 7.7^{* * *}$ & 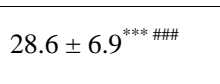 & $27.8 \pm 6.4^{+++}$ & $30.6 \pm 6.5$ & $27.4 \pm 5.3^{* *}$ & 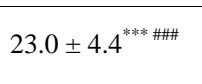 \\
\hline 3WDT (m) & $390.0 \pm 58.7$ & $403.3 \pm 58.9$ & $390.7 \pm 38.9$ & $353.7 \pm 68.0^{* * * \# \#}$ & $350.6 \pm 34.1^{+++}$ & $351.1 \pm 34.6$ & $358.2 \pm 31.6$ & $338.4 \pm 34.4^{\#}$ \\
\hline $\mathrm{LE}(\mathrm{n}-\mathrm{m})$ & $630.1 \pm 137.0$ & $669.3 \pm 130.8$ & $622.8 \pm 136.5$ & $537.7 \pm 108.3^{* * * \# \#}$ & $409.2 \pm 106.3^{+++}$ & $430.1 \pm 118.8$ & $420.3 \pm 91.1$ & $352.6 \pm 81.7^{* * * \# \#}$ \\
\hline
\end{tabular}

SD = Standard Deviation; CRT = chair-rising test; 3WDT = 3-min walk distance test; LE = leg extension; $\mathrm{V}_{\mathrm{O} 2 \mathrm{max}}=$ maximum oxygen uptake. 


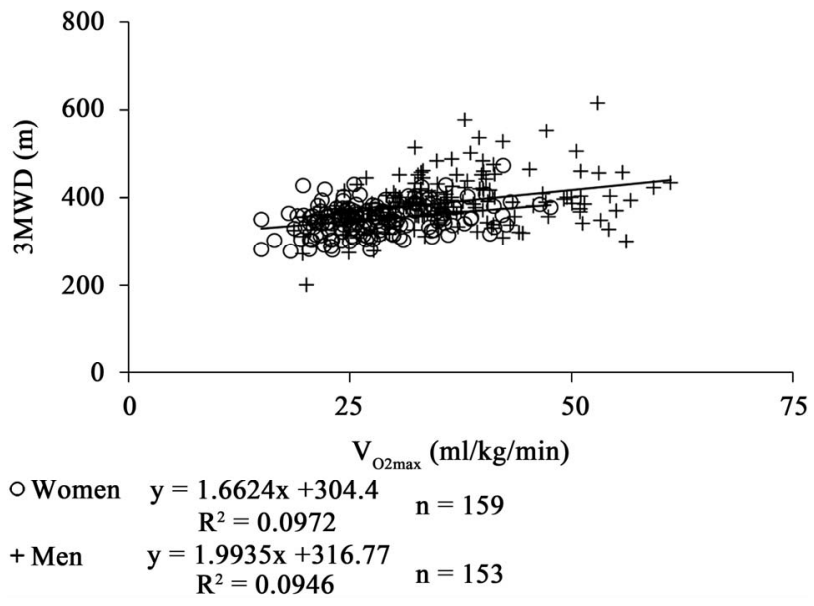

Figure 1. Relationship between $3 \mathrm{WTD}$ and $\mathrm{V}_{\mathrm{O} 2 \max }$ in the men ( $\mathrm{n}$ $=153)$ and women $(n=159)$. The 3MWD was to walk as many lengths as possible in three minutes. $\mathrm{V}_{\mathrm{O} 2 \max }$ was to until subject could not maintain the required pedaling frequency (60 rpm).

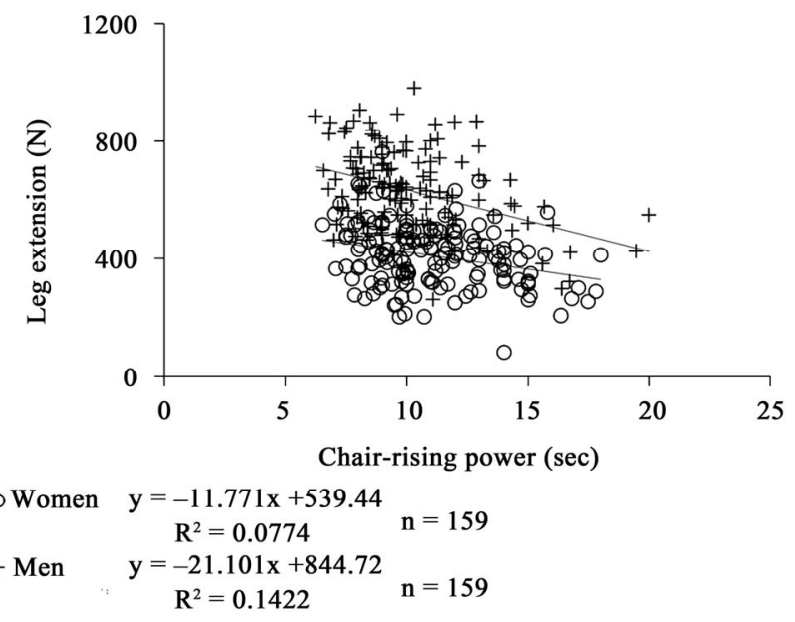

Figure 2. Relationship between LE and CRT in the men ( $\mathrm{n}=$ $153)$ and women $(n=159)$. The LE was to the highest in four times in total the right and left two times value. Chair-rising times from the initial seated position to the final seated position after completing ten stands were the test measure.

A remarkable decline, however, was observed in the performance variables (muscle strength and aerobic capacity) assessed by the CRT and by 3WDT. The CRT was measured power during an activity which involves raising the centre of gravity. Several studies have found that performance in the CRT is a strong predictor of incident disability, mortality, falls, hospitalization and health care resources consumption. Hence, CRT can be regarded as an indicator of physical performance at old age. In the present study, both in men and women were observed for the correlation between 3WDT and $\mathrm{V}_{\text {O2peak, }}$ LES and CRT respectively, and the fact that this test can be a feasible method of providing the information for muscle power and aerobic fitness, possibly avoiding the need for a maximal stress test.
Significant correlations among all the functional mobility tests in the older group indicate that older adults who performed poorly in one test were likely to perform poorly in all the other tests. The results from the present study, the functional mobility tests of 3WDT and CRT were found to give an idea of the physical decline with age in fit elderly without any maximal exercise stress.

In conclusion, first, this study provides significant agerelated differences in performance were found in tests of coordinated the $\mathrm{V}_{\text {O2peak }}$, 3WDT, LES, and CRT, with older women performing worse than older men in all tests. Secondly, this study shows that 3WDT and CRT can be a feasible method of providing the information for muscle power and aerobic fitness, possibly avoiding the need for a maximal stress test.

\section{Limit}

As the correlation coefficient between $3 \mathrm{WTD}$ and $\mathrm{V}_{\text {O2peak }}$, LES and CRT were low $(r=0.28-0.38)$, an error may occur. Accordingly, this study shows that 3WDT and CRT as estimate method for aerobic fitness and muscle power can be a feasible, if we measure many people as method briefly and in safety.

\section{REFERENCES}

[1] Guralnik, J.M., Ferrucci, L., Pieper, C.F., Leveille, S.G., Markides, K.S., Ostir, G.V., Studenski, S., Berkman, L.F. and Wallace, R.B. (2000) Lower extremity function and subsequent disability: Consistency across studies, predictive models, and value of gait speed alone compared with the short physical performance battery. Journals of Gerontology Series A: Biological Sciences and Medical Sciences, 55, M221-M231. doi:10.1093/gerona/55.4.M221

[2] Guralnik, J.M., Ferrucci, L., Simonsick, E.M., Salive, M.E. and Wallace, R.B. (1995) Lower-extremity function in persons over the age of 70 years as a predictor of subsequent disability. The New England Journal of Medicine, 332, 556-561. doi:10.1056/NEJM199503023320902

[3] Guralnik, J.M., Simonsick, E.M., Ferrucci, L., Glynn, R.J., Berkman, L.F., Blazer, D.G., Scherr, P.A. and Wallace, R.B. (1994) A short physical performance battery assessing lower extremity function: Association with selfreported disability and prediction of mortality and nursing home admission. The Journals of Gerontology, 49, M85M94.

[4] Aoyagi, Y. and Shephard, R.J. (1992) Aging and muscle function. Sports Medicine, 14, 376-396. doi:10.2165/00007256-199214060-00005

[5] Harriss, D.J. and Atkinson, G. (2009) Ethical standards in sport and exercise science research. International Journal of Sports Medicine, 30, 701-702. doi:10.1055/s-0029-1237378

[6] Janssen, I., Katzmarzyk, P.T. and Ross, R. (2005) Body mass index is inversely related to mortality in older peo- 
ple after adjustment for waist circumference. Journal of the American Geriatrics Society, 53, 2112-2118. doi:10.1111/j.1532-5415.2005.00505.x

[7] Lord, S. and Ward, J. (1994) Age-associated differences in sensori-motor function and balance in community dwelling women. Age and Ageing, 23, 452-460. doi:10.1093/ageing/23.6.452

[8] Himann, J.E., Cunningham, D.A., Rechnitzer, P.A. and Paterson, D.H. (1988) Age-related changes in speed of walking. Medicine \& Science in Sports \& Exercise, 20, 161-166. doi:10.1249/00005768-198820020-00010

[9] Bohannon, R.W. (1997) Comfortable and maximum walking speed of adults aged 20 - 79 years: Reference values and determinants. Age and Ageing, 26, 15-19. doi:10.1093/ageing/26.1.15

[10] Kerrigan, D.C., Todd, M.K., Della, C.U., Lipsitz, L.A. and Collins, J.J. (1998) Biomechanical gait alterations independent of speed in the healthy elderly: Evidence for specific limiting impairments. Archives of Physical Medicine and Rehabilitation, 79, 317-322. doi:10.1016/S0003-9993(98)90013-2

[11] Tiedemann, A., Sherrington, C. and Lord, S.R. (2005) Physiological and psychological predictors of walking speed in older community-dwelling people. Gerontology, 51, 390-395. doi:10.1159/000088703

[12] Tiedemann, A.C., Sherrington, C. and Lord, S.R. (2007) Physical and psychological factors associated with stair negotiation performance in older people. Journals of Gerontology Series A: Biological Sciences and Medical Sciences, 62, 1259-1265. doi:10.1093/gerona/62.11.1259
[13] Myers, A.M., Powell, L.E., Maki, B.E., Holliday, P.J., Brawley, L.R. and Sherk, W. (1996) Psychological indicators of balance confidence: Relationship to actual and perceived abilities. Journals of Gerontology Series A: Biological Sciences and Medical Sciences, 51, M37-M43. doi:10.1093/gerona/51A.1.M37

[14] Puthoff, M.L. and Nielsen, D.H. (2007) Relationships among impairments in lower-extremity strength and power, functional limitations, and disability in older adults. Physical Therapy, 87, 1334-1347. doi: $10.2522 / p t j .20060176$

[15] Tiedemann, A., Shimada, H., Sherrington, C., Murray, S.M. and Lord, S.R. (2008) The comparative ability of eight functional mobility tests for predicting falls in community-dwelling older people. Age and Ageing, 37, 430-435. doi:10.1093/ageing/afn100

[16] Cunningham, D.A., Rechnitzer, P.A., Pearce, M.E. and Donner, A.P. (1982) Determinants of self-selected walking pace across ages 19 to 66. The Journals of Gerontology, 37, 560-564.

[17] Lord, S.R., Tiedemann, A., Chapman, K., Munro, B., Murray, S.M. and Sherrington, C. (2005) The effect of an individualized fall prevention program on fall risk and falls in older people: A randomized, controlled trial. Journal of the American Geriatrics Society, 53, 12961304. doi:10.1111/j.1532-5415.2005.53425.X

[18] Samson, M., Meeuwsen, I., Crowe, A., Dessens, J., Duursma, S. and Verhaar, H. (2000) Relationships between physical performance measures, age, height and body weight in healthy adults. Age and Ageing, 29, 235242. doi:10.1093/ageing/29.3.235 